\title{
Impact simulation by an Eulerian model for interaction of multiple elastic-plastic solids and fluids
}

\author{
Sarah Hank, S.L. Gavrilyuk ${ }^{\dagger}$ Nicolas Favrie ${ }^{\ddagger}$ and Jacques Massoni ${ }^{\S}$
}

June 6, 2016

\begin{abstract}
A multiphase Eulerian formulation for the interaction of visco-plastic compressible solids and compressible fluids is proposed. The plasticity effects in solids are described by relaxation terms in the governing equations which are compatible with the Von Mises yield criterion. The visco-plastic model is validated on available experimental data in a range of the impact velocity : a high velocity symmetric rod-on-rod impact experiments, as well as low velocity impacts of jelly-like materials. A good agreement between numerical and experimental results is found.
\end{abstract}

\section{Introduction}

Solid-fluid interactions in the case of extreme deformations appear in many industrial applications (blast effects on structures, hypervelocity impacts,...). This kind of problems may involve high pressures and strain rates as well as a high density ratio. The hyperelastic models $[13,17,15,21,26,20,7]$ for which the stress tensor is defined in terms of a stored energy function are well adapted to treat accurately such problems. The hyperelastic models are conservative by construction. They are also objective and thermodynamically consistent. In this paper, a multi-component hyperelastic Eulerian formulation is used to compute several impact test cases [16]. The modelling is based on a 'diffuse interfaces method' which was developed for multi-component fluids $[1,28,28]$ and generalized to the case of interaction of multiple solids and fluids $[8,23]$. Relaxation terms for an accurate description of plastic transformations proposed in solids in [7] have been added. No hardening parameter is used to deal with the evolution of the yield strength.

The paper is organized as follows. In Section 2, the mathematical model is presented. In Section 3 , the numerical method is briefly described. Two test cases are studied in Section 4. In particular, a symmetric copper rod impact is computed and compared to the experimental data provided in [9]. Then, a low velocity clay suspension impact is studied and compared to the experimental results obtained in [19].

\section{Viscoplastic model}

\subsection{Eulerian multi-component formulation of hyperelasticity}

Hyperelasticity models have been intensively studied in the past few years $[13,15,14,2,21,17,20$, $26,12,25,3]$. In this paper, we consider a modified conservative formulation adapted to the case of

\footnotetext{
*Aix-Marseille Université, C.N.R.S. U.M.R. 7343, IUSTI, 5 rue E. Fermi, 13453 Marseille Cedex 13 France, hank.sarah@gmail.com

${ }^{\dagger}$ Corresponding author, Aix-Marseille Université, C.N.R.S. U.M.R. 7343, IUSTI, 5 rue E. Fermi, 13453 Marseille Cedex 13 France, sergey.gavrilyuk@univ-amu.fr

¥Aix-Marseille Université, C.N.R.S. U.M.R. 7343, IUSTI, 5 rue E. Fermi, 13453 Marseille Cedex 13 France, nicolas.favrie@univ-amu.fr

$\S$ Aix-Marseille Université, C.N.R.S. U.M.R. 7343, IUSTI, 5 rue E. Fermi, 13453 Marseille Cedex 13 France, jacques.massoni@univ-amu.fr
} 
isotropic solids. The Eulerian formulation of the multi-component hyperelasticity proposed in $[5,8,23]$ is considered. The numerical algorithm for solving this model is based on the generalization of the discrete equations method developed earlier for multi-component fluids in [1, 27, 28] and multi-component solids $[16]$.

As we deal with non-equilibrium flows, each component admits its own equation of state with its own stress tensor. This approach allows us to treat configurations involving several solids and fluids. The discrete equations are obtained by integrating the conservation laws over a multiphase control volume. The general model is written hereafter for the phase $k$ (in 1D case for the sake of simplicity).

$$
\left\{\begin{array}{l}
\frac{\partial \alpha_{k}}{\partial t}+u_{I} \frac{\partial \alpha_{k}}{\partial x}=0 \\
\frac{\partial(\alpha \rho)_{k}}{\partial t}+\frac{\partial(\alpha \rho u)_{k}}{\partial x}=0 \\
\frac{\partial(\alpha \rho u)_{k}}{\partial t}+\frac{\partial\left(\alpha \rho u^{2}-\alpha \sigma_{11}\right)_{k}}{\partial x}=-\sigma_{11, I} \frac{\partial \alpha_{k}}{\partial x}, \\
\frac{\partial(\alpha \rho)_{k}}{\partial t}+\frac{\partial(\alpha \rho u v)_{k}}{\partial x}+\frac{\partial\left(-\alpha \sigma_{12}\right)_{k}}{\partial x}=-\sigma_{12, I} \frac{\partial \alpha_{k}}{\partial x}, \\
\frac{\partial(\alpha \rho w)_{k}}{\partial t}+\frac{\partial(\alpha \rho u)_{k}}{\partial x}+\frac{\partial\left(-\alpha \sigma_{13}\right)_{k}}{\partial x}=-\sigma_{13, I} \frac{\partial \alpha_{k}}{\partial x}, \\
\frac{\partial(\alpha \rho E)_{k}}{\partial t}+\frac{\partial\left(\alpha \rho E u-\alpha \sigma_{11} u-\alpha \sigma_{12} v-\alpha \sigma_{13} w\right)_{k}}{\partial x}=-\left(\sigma_{11, I} u_{I}+\sigma_{12, I} v_{I}+\sigma_{13, I} w_{I}\right) \frac{\partial \alpha_{k}}{\partial x} \\
\frac{\partial\left(\alpha a^{\beta}\right)_{k}}{\partial t}+\frac{\partial\left(\alpha a^{\beta} u\right)_{k}}{\partial x}+\left(\alpha b^{\beta}\right)_{k} \frac{\partial v_{k}}{\partial x}+\left(\alpha c^{\beta}\right)_{k} \frac{\partial w_{k}}{\partial x}=0, \quad \beta=1,2,3 \\
\frac{\partial b_{k}^{\beta}}{\partial t}+u_{k} \frac{\partial b_{k}^{\beta}}{\partial x}=0, \quad \beta=1,2,3 \\
\frac{\partial c_{k}^{\beta}}{\partial t}+u_{k} \frac{\partial c_{k}^{\beta}}{\partial x}=0, \quad \beta=1,2,3 .
\end{array}\right.
$$

Here, for $k^{t h}$ phase: $\alpha_{k}$ is the volume fraction, $\rho_{k}$ is the phase density, $\mathbf{u}_{k}=\left(u_{k}, v_{k}, w_{k}\right)^{T}$ is the velocity field, $\boldsymbol{\sigma}_{k}$ is the stress tensor:

$$
\boldsymbol{\sigma}_{k}=\mathbf{S}_{k}-p_{k} \mathbb{I},
$$

where $\mathbf{S}_{k}$ is the deviatoric part of the stress tensor and $p_{k}$ is the thermodynamical pressure. As the model belongs to the class of hyperelastic models, the stress tensor can be expressed as the variation of the internal energy (their exact expressions are given in the following subsection). The evolution equations of hyperelasticity are written for deformation measures (in particular, for the Finger tensor defined below). $E_{k}$ is the total energy associated to the phase $k$ and is given by the following expression:

$$
E_{k}=\frac{\left\|\mathbf{u}_{k}\right\|^{2}}{2}+e_{k}\left(\eta_{k}, \mathbf{G}_{k}\right)
$$

where $\eta_{k}$ is the entropy of the phase $k$ and $\mathbf{G}_{k}$ is the Finger tensor. The exact expressions of $e_{k}\left(\eta_{k}, \mathbf{G}_{k}\right)$ in (3) will be given in the next subsection.

The variables with subscripts 'I' are the 'interface' variables. They are obtained directly when solving the Riemann problem. The model is thermodynamically consistent and satisfies the second principle of thermodynamics. The proof is not straightforward. Nevertheless, the thermodynamic consistency has been verified on numerical test cases (for example, a shock wave propagation in a media in presence of material interfaces). In the right hand side of the system (1), non conservative terms are present: these terms exist if the volume fraction gradient is non zero.

The geometric variables $a_{k}^{\beta}, b_{k}^{\beta}, c_{k}^{\beta}$ related to the deformation gradient will now be defined. To simplify the presentation, we will not further use in this section the subscript $k$ for unknowns. Let us define the Finger tensor $\mathbf{G}$ as the inverse of the left Cauchy-Green tensor $\mathbf{B}$ : $\mathbf{G}=\mathbf{B}^{-1}$. The Finger tensor can also be expressed in the form:

$$
\mathbf{G}=\sum_{\beta=1}^{3} \mathbf{e}^{\beta} \otimes \mathbf{e}^{\beta}, \quad \mathbf{e}^{\beta}=\left(a^{\beta}, b^{\beta}, c^{\beta}\right)^{T}, \quad \mathbf{e}^{\beta}=\nabla X^{\beta}, \quad \beta=1,2,3, \quad \mathbf{F}^{-T}=\left(\mathbf{e}^{1}, \mathbf{e}^{2}, \mathbf{e}^{3}\right) .
$$


Here $X^{\beta}$ are the Lagrangian coordinates, the gradient is taken with respect to the Eulerian coordinates, $\mathbf{F}$ is the deformation gradient. In the next subsection the equation of state is presented, allowing the system closure. Different relaxation phenomena can easily be added into the model (pressure and velocity relaxation, phase transitions...).

\subsection{System closure}

The closure of the system is performed by using an equation of state presented in a separable form [11]:

$$
e(\eta, \mathbf{G})=e^{h}(\rho, \eta)+e^{e}(\mathbf{g}), \mathbf{g}=\frac{\mathbf{G}}{|\mathbf{G}|^{1 / 3}},
$$

where $|\mathbf{G}|$ denotes the determinant of the tensor $\mathbf{G}$. This formulation has been used in particular in $[5,8,4,24]$. With such a formulation, the pressure is determined only by the hydrodynamic part of internal specific energy $e^{h}(\rho, \eta)$. The deviatoric part of the stress tensor can be expressed using the shear part of the specific internal energy $e^{e}(\mathbf{g})$. The hydrodynamic part of the energy satisfies the Gibbs identity:

$$
\theta d \eta=d e^{h}+p d \tau
$$

where $\tau$ is the specific volume $(\tau=1 / \rho)$ and $\theta$ is the temperature. The expression of the deviatoric part of the stress tensor $S$ is:

$$
S=-2 \rho \frac{\partial e^{e}}{\partial \mathbf{G}} \mathbf{G} .
$$

The hydrodynamic part of the internal specific energy is taken as the stiffened gas equation of state:

$$
e^{h}(\rho, p)=\frac{p+\gamma p_{\infty}}{\rho(\gamma-1)}
$$

In [10], a family of rank-one convex stored energies for isotropic compressible solids with a single parameter (denoted by $\tilde{a}$ ) is proposed:

$$
e^{e}(\mathbf{G})=\frac{\mu}{4 \rho_{0}}\left(\frac{1-2 \tilde{a}}{3} j_{1}^{2}+\tilde{a} j_{2}+3(\tilde{a}-1)\right), \quad j_{m}=\operatorname{tr}\left(\mathbf{g}^{m}\right), m=1,2,3 .
$$

Here, $\mu$ is the shear modulus of the considered material and $\rho_{0}$ is the reference density. Using the criterion proposed in $[22,10]$, it has been proven that with the equations of state (5) and (6), the equations are hyperbolic for any $\tilde{a}$ such that $-1 \leq \tilde{a} \leq 0.5$. The relation (6) involves the following expression for the deviatoric part:

$$
\mathbf{S}=-\mu \frac{\rho}{\rho_{0}}\left(\frac{1-2 \tilde{a}}{3} j_{1}\left\{\mathbf{g}-\frac{j_{1}}{3} \mathbb{I}\right\}+\tilde{a}\left\{\mathbf{g}^{2}-\frac{j_{2}}{3} \mathbb{I}\right\}\right) .
$$

One can notice that for the value $\tilde{a}=-1$, the equation of state describes neo-Hookean solids. Its expression is the following:

$$
e^{e}(\mathbf{G})=\frac{\mu}{4 \rho_{0}}\left(j_{1}^{2}-j_{2}-6\right) .
$$

The energy (8) is, in particular, suitable for the description of jelly-type materials. In the case of metals, the value $\tilde{a}=0.5$ can be chosen, where the equation of state becomes:

$$
e^{e}(\mathbf{G})=\frac{\mu}{8 \rho_{0}}\left(j_{2}-3\right)
$$




\subsection{Viscoplasticity modelling}

An important class of hyperbolic models describing the plastic behavior of materials under large stresses has been proposed, for example in $[13,15,14,2]$. An extension of this approach has been proposed in $[5,7,6,23]$ to include material yield criteria (Von Mises). The relaxation terms are constructed in such a way that they are compatible with the mass conservation law and consistent with the second law of thermodynamics. The Von Mises yield limit is reached at the end of the relaxation step. The built model belongs to Maxwell type model, where the intensity of the shear stress decreases during the relaxation.

We use the formulation proposed in [7]. The governing equations for $\mathbf{e}^{\beta}$ are now written as follows:

$$
\frac{D \mathbf{e}^{\beta}}{D t}+\left(\frac{\partial \mathbf{u}}{\partial \mathbf{x}}\right)^{T} \mathbf{e}^{\beta}=-\frac{1}{\tau_{r e l}} \mathbf{R e}^{\beta},
$$

where $\tau_{\text {rel }}$ corresponds to a relaxation time and $\mathbf{R}$ is a symmetric tensor $\left(\mathbf{R}=\mathbf{R}^{T}\right)$. As the Finger tensor $\mathbf{G}$ is linked to the local cobasis $\mathbf{e}^{\beta}$, it is possible to write the governing equation for $\mathbf{G}$ by using (10).

$$
\frac{D \mathbf{G}}{D t}+\left(\frac{\partial \mathbf{u}}{\partial \mathbf{x}}\right)^{T} \mathbf{G}+\mathbf{G}\left(\frac{\partial \mathbf{u}}{\partial \mathbf{x}}\right)=-\frac{1}{\tau_{r e l}}(\mathbf{G R}+\mathbf{R G})
$$

In [7], an expression has been proposed for the tensor $\mathbf{R}$. This expression ensures the thermodynamic compatibility of the model:

$$
\mathbf{R}=-a \mathbf{S}
$$

with $\mathbf{S}$ being the deviatoric part of the stress tensor derived from (6):

$$
\mathbf{S}=-\mu \frac{\rho}{\rho_{0}}\left(\frac{1-2 \tilde{a}}{3} j_{1}\left\{\mathbf{g}-\frac{j_{1}}{3} \mathbb{I}\right\}+\tilde{a}\left\{\mathbf{g}^{2}-\frac{j_{2}}{3} \mathbb{I}\right\}\right) .
$$

The relaxation step is performed after the hyperbolic step: there is no space variation during the relaxation process. The derivative $D / D t$ should be replaced by the partial derivative with respect to time:

$$
\frac{D}{D t}=\frac{\partial}{\partial t}
$$

In the following, we write $\frac{\partial}{\partial t}=\frac{d}{d t}$. We have to solve the following relaxation equation for each cell:

$$
\frac{d \mathbf{G}}{d t}=\frac{a}{\tau_{r e l}}(\mathbf{G S}+\mathbf{S G})=\frac{2 a}{\tau_{r e l}}(\mathbf{G S}) .
$$

\subsubsection{Von Mises yield criterion}

The Von Mises criterion implies that the material starts to yield when the corresponding yield function (noted $f(\mathbf{S})$ ) becomes positive:

$$
f(\mathbf{S})=\mathbf{S}: \mathbf{S}-\frac{2}{3} \sigma_{Y}^{2},
$$

Here $\sigma_{Y}$ is the yield strength. When the yield function is negative, the material has an elastic behavior. In this case, the relaxation time $\tau_{\text {rel }}$ becomes infinite. If the yield function is positive we have to relax the deformations in such a way that at the end of the relaxation step, the yield surface is recovered.

The value of $a$ is taken as,

$$
a=\frac{1}{2(\mathbf{S}: \mathbf{S})^{1 / 2}}
$$


The following expression for the relaxation time is used [7]:

$$
\frac{1}{\tau_{r e l}}= \begin{cases}\frac{1}{\tau_{0}}\left(\frac{\mathbf{S}: \mathbf{S}-\frac{2}{3} \sigma_{Y}^{2}}{\sigma_{Y}^{2}}\right)^{n}, & \text { if } \frac{\mathbf{S}: \mathbf{S}-\frac{2}{3} \sigma_{Y}^{2}}{\sigma_{Y}^{2}}>0 \\ 0, & \text { if } \quad \frac{\mathbf{S}: \mathbf{S}-\frac{2}{3} \sigma_{Y}^{2}}{\sigma_{Y}^{2}} \leq 0 .\end{cases}
$$

The values of the characteristic time $\tau_{0}$ and the exponent $n$ will be chosen latter. This expression is analogous to the Odquist law [18].

\section{Numerical treatment}

It has been proven that system (1) is hyperbolic when closed by the equation of state (5) and (6) ([24], [10]). The full system admits 7 characteristic eigenfields corresponding to 2 longitudinal waves, 4 shear waves and a contact discontinuity. The resolution of the Riemann problem is not straightforward. In order to simplify the resolution of the Riemann problem, a numerical splitting is performed for the full system. This method has been proposed in [4]. The full system is split in three sub-models, each of them is hyperbolic. The first sub-system deals with the longitudinal waves and the contact discontinuity while other sub-systems deal with the shear waves. The numerical splitting simplifies the solution of the Riemann problem at each cell edges. Indeed, as each sub-model admits the propagation of three waves. An HLLC type Riemann solver can be considered to compute fluxes. The details of the splitting for the multi-component case and multi-dimensional case are presented in [16]. The three sub-models are written hereafter (in one dimensional case).

$$
\left\{\begin{array}{l}
\frac{\partial \alpha_{k}}{\partial t}+u_{I} \frac{\partial \alpha_{k}}{\partial x}=0 \\
\frac{\partial(\alpha \rho)_{k}}{\partial t}+\frac{\partial(\alpha \rho u)_{k}}{\partial x}=0 \\
\frac{\partial(\alpha \rho u)_{k}}{\partial t}+\frac{\partial\left(\alpha \rho u^{2}-\alpha \sigma_{11}\right)_{k}}{\partial x}=-\sigma_{11, I} \frac{\partial \alpha_{k}}{\partial x} \\
\frac{\partial(\alpha \rho)_{k}}{\partial t}+\frac{\partial(\alpha \rho u)_{k}}{\partial x}=0 \\
\frac{\partial(\alpha \rho w)_{k}}{\partial t}+\frac{\partial(\alpha \rho u)_{k}}{\partial x}=0 \\
\frac{\partial(\alpha \rho)_{k}}{\partial t}+\frac{\partial\left(\alpha \rho E u-\alpha \sigma_{11} u\right)_{k}}{\left.\partial t^{\beta}\right)_{k}}=-\sigma_{11, I} u_{I} \frac{\partial\left(\alpha \alpha_{k}{ }^{\beta} u\right)_{k}}{\partial x}=0, \quad \beta=1,2,3, \\
\frac{\partial(x)}{\partial t}+u_{k} \frac{\partial b_{k}^{\beta}}{\partial x}=0, \quad \beta=1,2,3, \\
\frac{\partial c_{k}^{\beta}}{\partial t}+u_{k} \frac{\partial c_{k}^{\beta}}{\partial x}=0, \quad \beta=1,2,3 .
\end{array}\right.
$$

System (16) deals with the longitudinal waves and the contact discontinuity. The variables with the subscript 'I' are used to identify the interface variables. These interface quantities are obtained by solving the Riemann problem. The longitudinal sound speed is given by the following expression:

$$
c_{k}^{L}=\sqrt{\left.\frac{\partial p_{k}}{\partial \rho_{k}}\right|_{\eta_{k}}-\frac{\partial S_{11 k}}{\partial \rho_{k}}-\frac{1}{\rho_{k}} \sum_{\beta=1}^{3} \frac{\partial S_{11 k}}{\partial a_{k}^{\beta}} a_{k}^{\beta}} .
$$


The sub-systems for transverse waves are:

$$
\left\{\begin{array} { l } 
{ \frac { \partial \alpha _ { k } } { \partial t } = 0 } \\
{ \frac { \partial ( \alpha \rho ) _ { k } } { \partial t } = 0 } \\
{ \frac { \partial ( \alpha \rho u ) _ { k } } { \partial t } = 0 } \\
{ \frac { \partial ( \alpha \rho v ) _ { k } } { \partial t } - \frac { \partial ( \alpha \sigma _ { 1 2 } ) _ { k } } { \partial x } = - \sigma _ { 1 2 _ { I } } \frac { \partial \alpha _ { k } } { \partial x } } \\
{ \frac { \partial ( \alpha \rho w ) _ { k } } { \partial t } = 0 } \\
{ \frac { \partial ( \alpha \rho ) _ { k } } { \partial t } + \frac { \partial ( - \alpha \sigma _ { 1 2 } v ) _ { k } } { \partial x } = - \sigma _ { 1 2 _ { I } } v _ { I } \frac { \partial \alpha _ { k } } { \partial x } } \\
{ \frac { \partial ( \alpha a ^ { \beta } ) _ { k } } { \partial t } + ( \alpha b ^ { \beta } ) _ { k } \frac { \partial v _ { k } } { \partial x } = 0 , \quad \beta = 1 , 2 , 3 } \\
{ \frac { \partial b _ { k } ^ { \beta } } { \partial t } = 0 , \quad \beta = 1 , 2 , 3 } \\
{ \frac { \partial c _ { k } ^ { \beta } } { \partial t } = 0 , \quad \beta = 1 , 2 , 3 }
\end{array} \quad \left\{\begin{array}{l}
\frac{\partial \alpha_{k}}{\partial t}=0 \\
\frac{\partial(\alpha \rho)_{k}}{\partial t}=0 \\
\frac{\partial(\alpha \rho u)_{k}}{\partial t}=0 \\
\frac{\partial(\alpha \rho v)_{k}}{\partial t}=0 \\
\frac{\partial(\alpha \rho w)_{k}}{\partial t}-\frac{\partial\left(\alpha \sigma_{13}\right)_{k}}{\partial x}=-\sigma_{13_{I}} \frac{\partial \alpha_{k}}{\partial x} \\
\frac{\partial(\alpha \rho)_{k}}{\partial t}+\frac{\partial\left(-\alpha \sigma_{13} w\right)_{k}}{\partial x}=-\sigma_{13_{I}} w_{I} \frac{\partial \alpha_{k}}{\partial x} \\
\frac{\partial\left(\alpha a^{\beta}\right)_{k}}{\partial t}+\left(\alpha c^{\beta}\right)_{k} \frac{\partial w_{k}}{\partial x}=0, \quad \beta=1,2,3 \\
\frac{\partial b_{k}^{\beta}}{\partial t}=0, \quad \beta=1,2,3 \\
\frac{\partial c_{k}^{\beta}}{\partial t}=0, \quad \beta=1,2,3
\end{array}\right.\right.
$$

The expressions of the transverse sound speeds are given hereafter:

$$
c_{k}^{t 1}=\sqrt{-\frac{1}{\rho_{k}} \sum_{\beta=1}^{3} \frac{\partial S_{12 k}}{\partial a_{k}^{\beta}} b_{k}^{\beta}}, \quad c_{k}^{t 2}=\sqrt{-\frac{1}{\rho_{k}} \frac{\partial S_{13 k}}{\partial a_{k}^{\beta}} c_{k}^{\beta}} .
$$

Both sub-systems (18) deal with the shear waves and can be solved simultaneously. The three submodels correspond to the continuous limit of the discrete models obtained by integrating the pure solid equations over a multiphase control volume. Each sub-system is hyperbolic (see [16] for details). The integration scheme is a first order finite volume Godunov type scheme. The fluxes must be calculated at each cell edges. To do this, a HLLC type solver is used [31].

After the hyperbolic step solving (16), (18), relaxation terms are treated. A fourth order RungeKutta scheme is considered because of the stiffness of the right-hand side terms. Two versions of the code have been developed: a 3D code and 2D axi-symmetric version, both are parallel. The parallelization is performed using the domain decomposition method and using the openMPI library (open source Message Passing Interface).

\section{Validations}

The aim of this section is the validation of the elastic-plastic model. Two test cases are considered in this section: high and low velocity impacts.

\subsection{Impact of a copper rod}

The plastic deformation induced by the normal impact of a rod is a classical problem of impact solid dynamics $([29,30])$. We use experimental data provided in [9], where a symmetric rod-on-rod impact has been studied. Symmetric rod-on-rod impact at velocity $V$ is equivalent to a "classical" impact at velocity $V / 2$. Symmetric impact allows us not to consider properties of the impacted surface which can be important in the case of the "classical" Taylor impact.

\subsubsection{Initial configuration}

The initial configuration is presented in Figure 1. A bi-dimensional axi-symmetric formulation of the equations is considered. The calculation domain is $105 \mathrm{~mm}$ high and $12 \mathrm{~mm}$ long and involves $2520 \times 288$ 

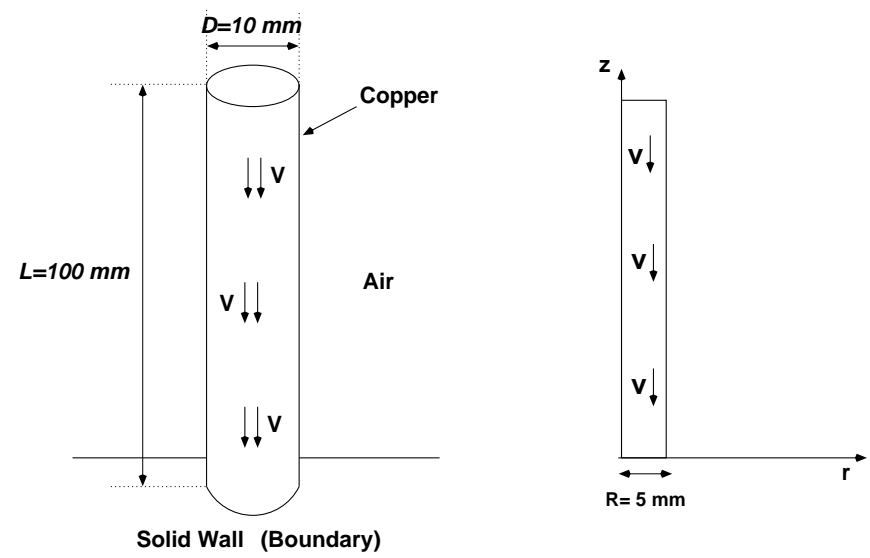

Figure 1: Studied configuration: the impact of a copper rod on a solid wall is performed. The impact velocity is $197.5 \mathrm{~m} / \mathrm{s}$. The rod diameter is $D=10 \mathrm{~mm}$ and the length $L=100 \mathrm{~mm}$.

cells. The final physical time is $368 \mu \mathrm{s}$. We are interested in the study of the temporal evolution of the shape of the copper rod. The numerical results are then compared to those of [9] wherein the authors measure the copper rod radius as a function of the distance from the impact interface.

Two materials are used for these computations: copper and air. Physical characteristics of both components are given in table 1 .

\begin{tabular}{|c|c|c|c|c|c|c|c|}
\hline Material & $\gamma$ & $P_{\infty}(G P a)$ & $\mu(G P a)$ & $\sigma_{Y}(M P a)$ & $\rho_{0}\left(\mathrm{~kg} / \mathrm{m}^{3}\right)$ & $\tau_{0}(s)$ & $n$ \\
\hline Copper & 4.54 & 29.9 & 60 & 450 & 8924 & $6.10^{-6}$ & 2 \\
\hline Air & 1.4 & 0 & - & - & 1.19 & - & - \\
\hline
\end{tabular}

Table 1: stiffened gas parameters and physical characteristics of copper and air.

The properties of the stiffened gas equation of state are determined by using the Russian Shock wave database www.ficp.ac.ru/rusbank/.

\subsubsection{Simulation results}

The available experimental data are given up to $68 \mu \mathrm{s}$. We have made the choice to perform the simulation until the stationary state would be reached. This allows us to measure the final dimensions of the rod (final length, final undeformed length) and especially to compare with the Taylor theory.

\subsubsection{Comparison with the experimental results}

The rod profiles obtained at different instants are compared to those given in [9], where the authors present high speed photograph of the rod-on-rod impact. The rod profiles are extracted at various time instants from the moment of impact, as a function of the distance from the impact interface. In Figure 2 , the experimental results are compared to the numerical ones. The numerical profiles are obtained by extracting the contours of copper volume fractions. The value 0.5 of these contours corresponds to the position of the copper/air interface.

The results presented in Figure 2 show a good agreement, particularly regarding the global shape of the rod. The rod radius at the impact is under-evaluated by the model, especially during the first instants, when the deformation is mainly located near the impact interface. The gap between 

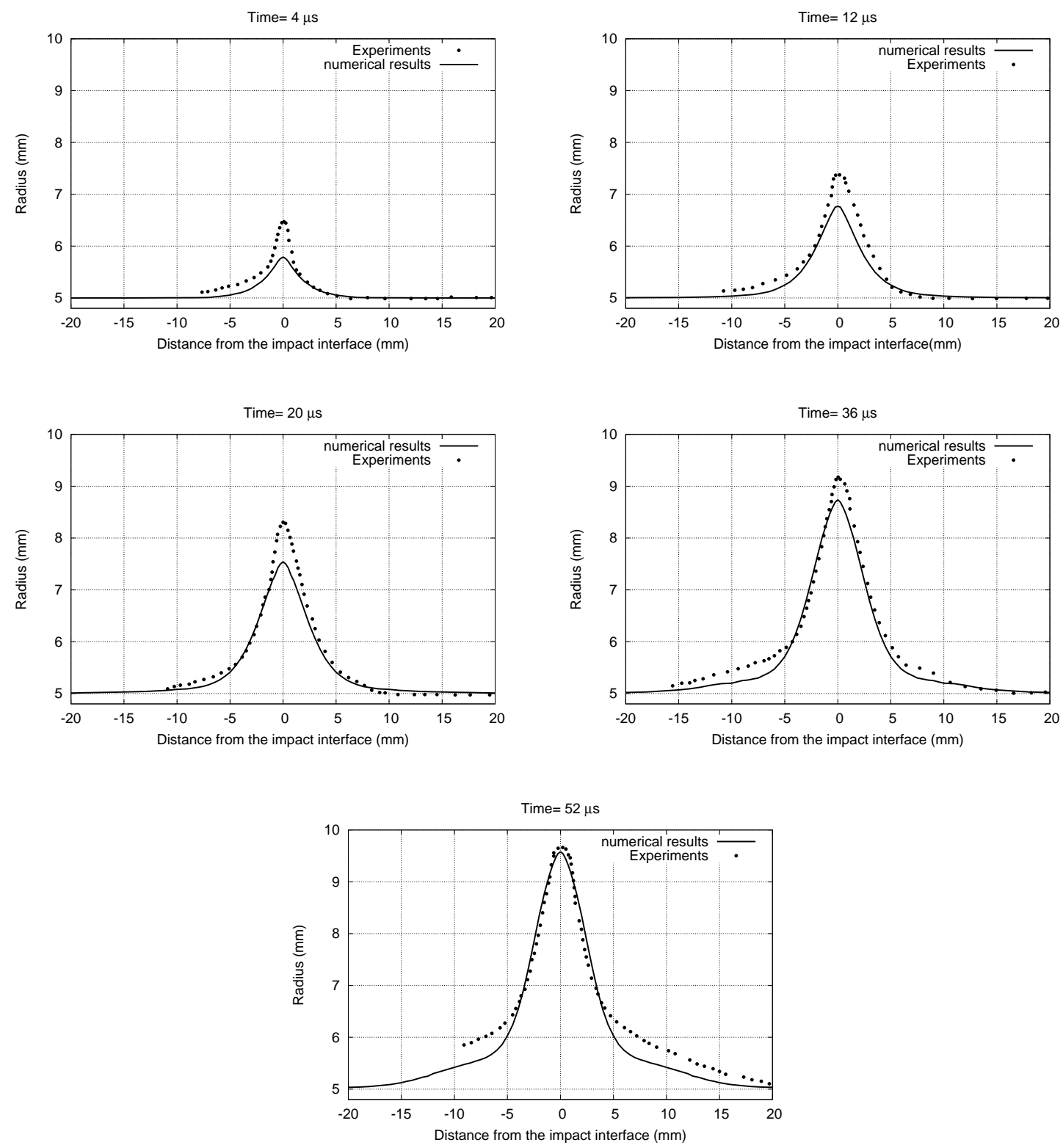

Figure 2: The copper rod radius is plotted as a function of the distance from the impact interface at several instants.

experimental results and numerical results decreases with time. The gap can be explained by the fact that we did not use work-hardening in the elastic-plastic model. Nevertheless, the error is quite small, just about $1 \mathrm{~mm}$. In Figure 3, the rod radius at the impact interface is plotted as a function of time as well as the rod total length. The final radius is reached after $100 \mu \mathrm{s}$, its value is $10.74 \mathrm{~mm}$, the total rod length tends to the value of $73.1 \mathrm{~mm}$.

The qualitative evolution of the copper rod profile is presented in order to appreciate the deformation induced by the impact. The Figure 4 shows the deformation of the copper rod due to the impact at different instants. It allows us to notice the appearance of the 'shoulders' on the rod shape (see the rod shape at instant $128 \mu \mathrm{s}$ in Figure 4). The rod shape tends to a stationary state after about $200 \mu s$. 

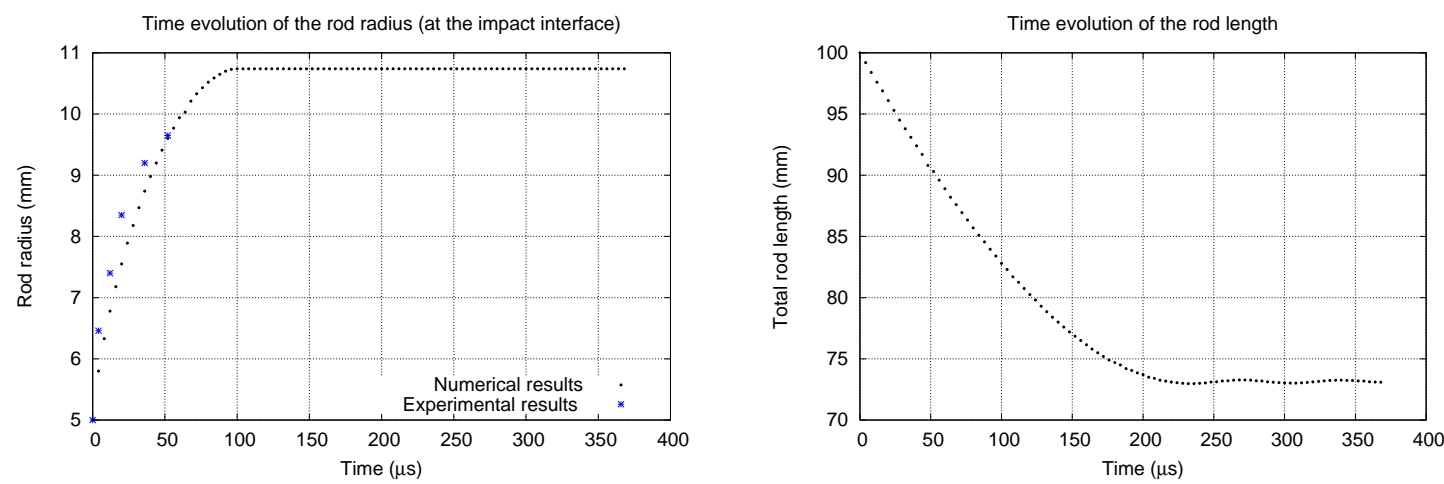

Figure 3: Time evolution of the rod radius at the impact interface between $0 \mu s$ and $368 \mu s$.
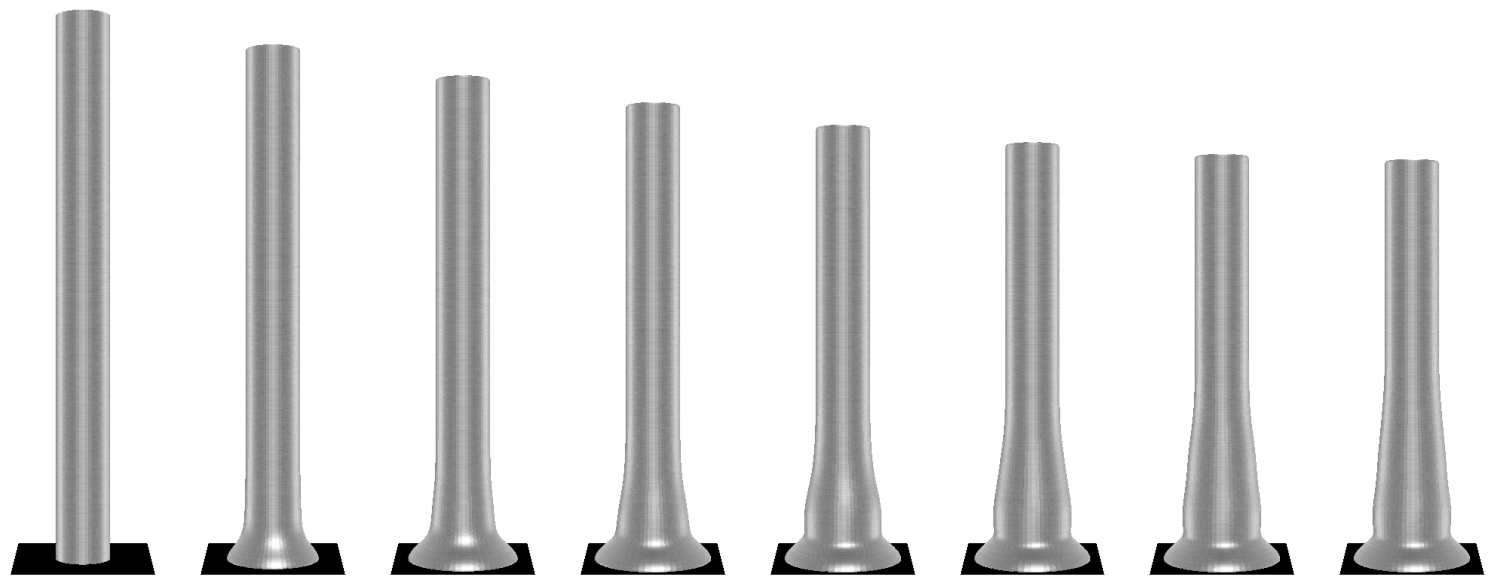

Figure 4: The copper rod is presented at several instants from the moment of the impact, $0 \mu \mathrm{s}, 32 \mu \mathrm{s}$, $64 \mu s, 96 \mu s, 128 \mu s, 160 \mu s, 192 \mu s$ and $320 \mu s$. The shape of the rod changes during the impact and tends to a stationary state. The formation of "shoulders" (of a new "inflection" point at the rod shape) can clearly be observed at the instant $128 \mu \mathrm{s}$.

It is interesting to compare the resulting final dimensions with the Taylor analysis [30]. Let $L_{1}$ be the final length of the rod and $X$ the undeformed rod length. The Taylor theory links the final rod dimensions to features of the impact:

$$
\frac{\sigma_{Y}}{\rho_{p} V^{2}}=\frac{L-X}{2\left(L-L_{1}\right)} \frac{1}{L / X}
$$

The simulation gives the following values : $L_{1}=73.1 \mathrm{~mm}$ and $X=39 \mathrm{~mm}$. It gives us :

$$
\frac{L-X}{2\left(L-L_{1}\right)} \frac{1}{L / X} \approx 1.04 .
$$

The gap between the theoretical value and the calculated one is equal to 6.8 per cent. 


\subsection{Impact of a jelly-like material}

\subsubsection{Studied configuration}

In this simulation, a sample of clay suspension of diameter $D$ normally impacts a flat rigid surface. This kind of impact has been studied, in particular, in [19]. Experiments were made on different surface types (smooth glass surface and super hydrophobic surface). In this paper, we extract the results associated to the bentonite impacting a smooth glass surface. In particular, we are interested in the final diameter of the impacting drop. The studied configuration is presented in Figure 5. Let us define
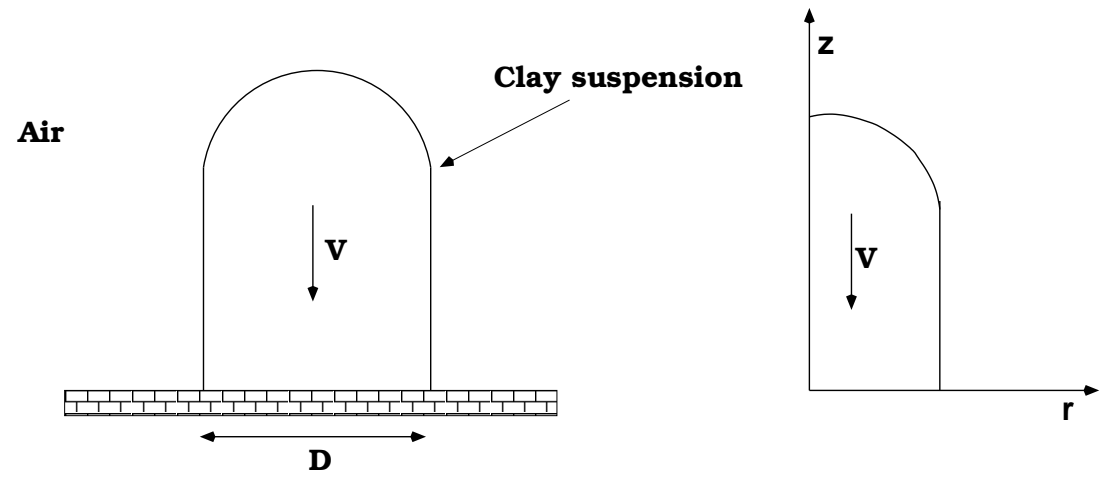

Figure 5: Studied configuration: the impact of a clay suspension cylinder at the velocity $\mathbf{V}$.

$L_{0}$ as the diameter of the equivalent sphere of the same volume. Two materials are present in this this configuration : the clay suspension cylinder (bentonite) and surrounded air. The corresponding material parameters are given in Table 2.

\begin{tabular}{|c|c|c|c|c|c|c|c|}
\hline Material & $\gamma$ & $P_{\infty}(G P a)$ & $\mu(P a)$ & $\sigma_{Y}(P a)$ & $\rho_{0}\left(\mathrm{~kg} / \mathrm{m}^{3}\right)$ & $\tau_{0}(s)$ & $n$ \\
\hline Bentonite & 2.35 & 0.005 & 2400 & 68 & 1090 & $10^{-6}$ & 0.5 \\
\hline Air & 1.4 & 0 & - & - & 1.19 & - & - \\
\hline
\end{tabular}

Table 2: stiffened gas parameters and features of the clay suspension and air.

The initial diameter of the cylinder is equal to $14 \mathrm{~mm}$. The associated value of $L_{0}$ is $15.08 \mathrm{~mm}$. The experimental results show a quasi-linear behavior of the maximal spread factor with respect to the impact velocity. The aim is then to see if the elastic-plastic model can reproduce this evolution.

\subsubsection{Numerical results}

Several simulations has been performed with various values of the impact velocity $(1 \mathrm{~m} / \mathrm{s}, 2 \mathrm{~m} / \mathrm{s}, 3 \mathrm{~m} / \mathrm{s}$, $4 \mathrm{~m} / \mathrm{s}$ ). The results of these computations are summarized in Figure 6 where the numerical results are compared to those of [19]. The numerical results are in a good agreement with the experimental ones. The points corresponding to the numerical results follow a straight line.

Let us define the time $T_{0}$ such that,

$$
T_{0}=\frac{L_{0}}{V_{0}}
$$

Here $L_{0}$ is the characteristic dimension of the droplet, and $V_{0}$ is the impact velocity. The dimensionless time is then given by $t / T_{0}$, where $t$ corresponds to the physical time. Figure 7 shows the qualitative comparison of the numerical results with the experimental ones at several time instants $\left(t / T_{0}=0.07, t / T_{0}=0.3 t / T_{0}=0.6, t / T_{0}=0.8\right)$. 


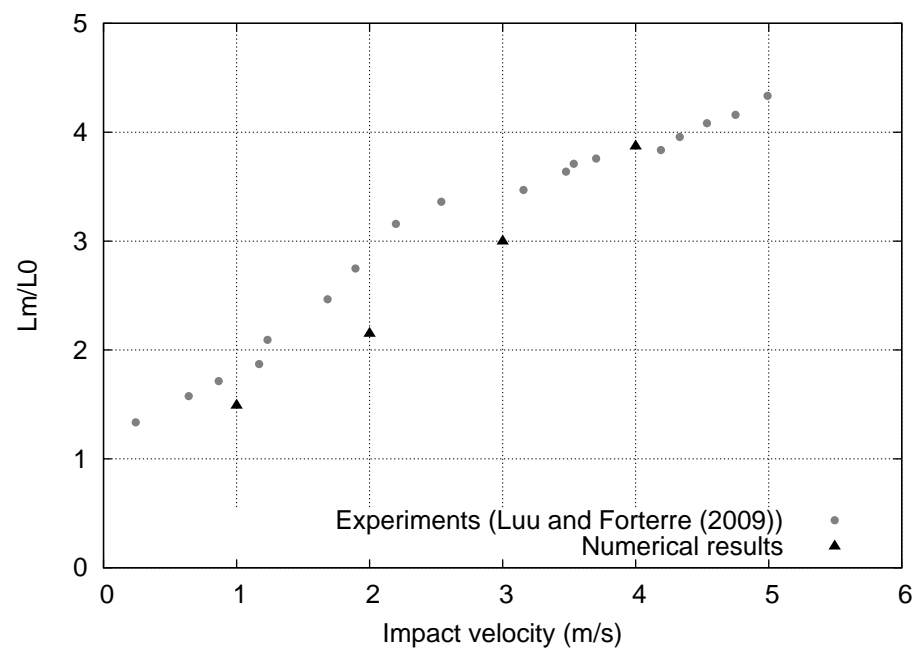

Figure 6: The maximal spread factor $L_{m} / L_{0}$ as a function of the impact velocity in the case of a bentonite drop impacting smooth glass surface (comparison with the experimental data of [19]).
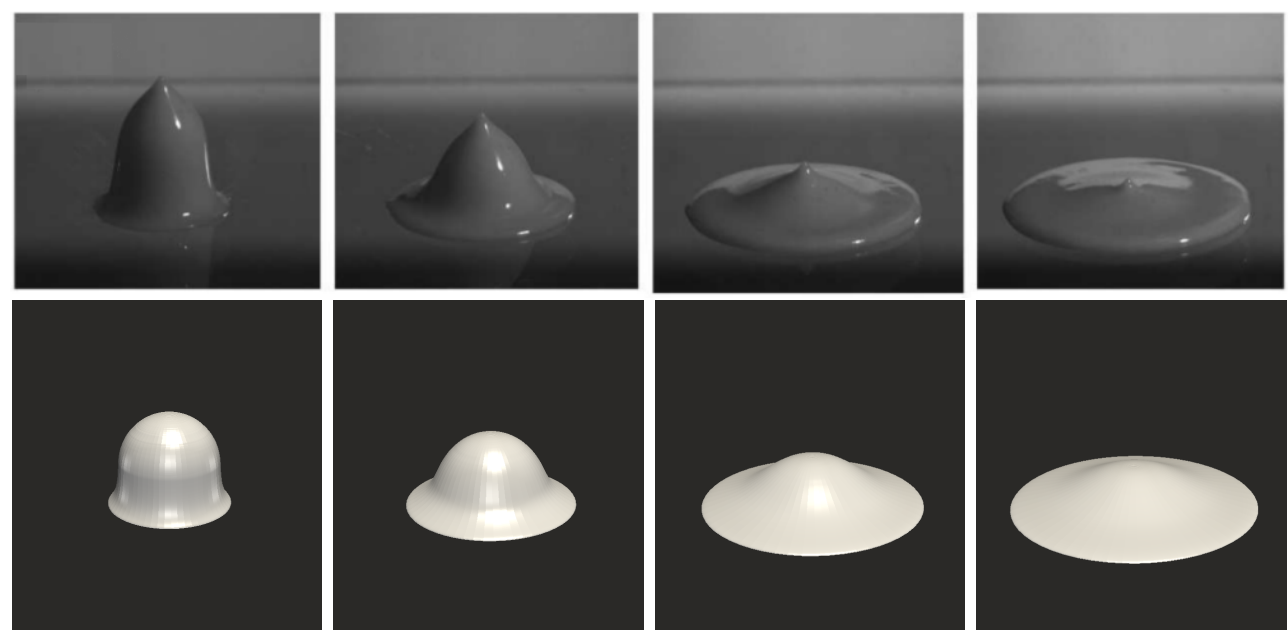

Figure 7: Comparison between the experimental results (at the top) with the numerical results (at the bottom) for several time instants of $t / T_{0}\left(t / T_{0}=0.07, t / T_{0}=0.3 t / T_{0}=0.6, t / T_{0}=0.8\right)$. The velocity impact is equal to $2 \mathrm{~m} / \mathrm{s}$.

The initial shape of the 'numerical' drop (see Figure 5) is not exactly the same one compared to the real drop. The real drop is generated by a syringe driver and falls then freely under gravity. This can explain a 'pointed' crest observable on the real drop. The dynamic behaviour of the bentonite drop is well reproduced by the simulation. A good agreement with the experimental results can be observed, both for the maximal spread factor (Figure 6) and for the form of the drop (Figure 7).

\section{Conclusion}

A visco-plastic Eulerian hyperbolic model is proposed. A simulation tool has been was developed to model simultaneously an arbitrary number of materials of different nature (fluids and solids). It is 
validated on impact experiments involving impact velocities varying from $1 \mathrm{~m} / \mathrm{s}$ to $200 \mathrm{~m} / \mathrm{s}$. Very different materials were considered: clay suspension and copper. The numerical solution is in a good agreement with the experimental data. The developed numerical model is also able to describe more complex phenomena like cracks formation and spallation in materials. These results will be presented in future publications.

\section{Acknowledgements}

We thank Y. Forterre for providing us the experimental data, and P. Le Tallec and P. H. Maire for useful discussion. This work was partially supported by l'Agence Nationale de la Recherche, France (grant numbers ANR-14-ASTR-0016-01, ANR-11-LABX-0092, and ANR-11-IDEX-0001-02). 


\section{References}

[1] R. Abgrall and R. Saurel. Discrete equations for physical and numerical compressible multiphase mixtures. Journal of Computational Physics, 186(2):361-396, 2003.

[2] P.T. Barton, D. Drikakis, and E.I. Romenski. An Eulerian finite-volume scheme for large elastoplastic deformations in solids. International journal for numerical methods in engineering, 81(4):453484, 2010.

[3] A. de Brauer, A. Iollo, and T. Milcent. A Cartesian scheme for compressible multimaterial models in 3D. Journal of Computational Physics, 313:121-143, 2016.

[4] N. Favrie, S.L. Gavrilyuk, and S. Ndanou. A thermodynamically compatible splitting procedure in hyperelasticity. Journal of Computational Physics, 270:300-324, 2014.

[5] N. Favrie, S.L. Gavrilyuk, and R. Saurel. Solid-fluid diffuse interface model in cases of extreme deformations. Journal of Computational Physics, 228(16):6037-6077, 2009.

[6] N. Favrie and Gavrilyuk S.L. Dynamics of shock waves in elastic-plastic solids. ESAIM: Proceedings, 33(1947):50-67, 2011.

[7] N. Favrie and Gavrilyuk S.L. Mathematical and numerical model for nonlinear viscoplasticity. Philosophical Transactions of the Royal Society of London A: Mathematical, Physical and Engineering Sciences, 369(1947):2864-2880, 2011.

[8] N. Favrie and Gavrilyuk S.L. Diffuse interface model for compressible fluid-compressible elasticplastic solid interaction. Journal of Computational Physics, 231(7):2695-2723, 2012.

[9] L.C. Forde, W.G. Proud, and S.M. Walley. Symmetrical Taylor impact studies of copper. Proceedings of the Royal Society of London A: Mathematical, Physical and Engineering Sciences, 465:769-790, 2009.

[10] S. Gavrilyuk, S. Ndanou, and S. Hank. An example of a one-parameter family of rank-one convex stored energies for isotropic compressible solids. Journal of Elasticity, 124:133-141, 2016.

[11] S.L. Gavrilyuk, N. Favrie, and R. Saurel. Modelling wave dynamics of compressible elastic materials. Journal of Computational Physics, 227(5):2941-2969, 2008.

[12] N. Ghaisas, A. Subramaniam, and S.K. Lele. High-order Eulerian methods for elastic-plastic flow in solids and coupling with fluid flows. Annual Research Briefs, 2015.

[13] S.K. Godunov. Elements of continuum mechanics. Moscow, Nauka, 1, 1978.

[14] S.K Godunov and I.M. Peshkov. Thermodynamically consistent nonlinear model of elastoplastic Maxwell medium. Computational mathematics and mathematical physics, 50(8):1409-1426, 2010.

[15] S.K. Godunov and E.I. Romenskii. Elements of continuum mechanics and conservation laws. Kluwer Academic/Plenum Publishers, 2003.

[16] S. Hank, N. Favrie, and Massoni J. Modeling hyperelasticity in non equilibrium multiphase flows. Hal, https://hal.archives-ouvertes.fr/hal-01316648.

[17] G. Kluth and B. Després. Perfect plasticity and hyperelastic models for isotropic materials. Continuum Mechanics and Thermodynamics, 20(3):173-192, 2008.

[18] J. Lemaitre, J.L. Chaboche, A. Benallal, and R. Desmorat. Mécanique des matériaux solides. Dunod, 2009.

[19] L.H. Luu and Y. Forterre. Drop impact of yield-stress fluids. Journal of Fluid Mechanics, 632:301327, 2009. 
[20] L.A. Merzhievsky and A.D. Resnyansky. The role of numerical simulation in the study of highvelocity impact. International journal of impact engineering, 17(4):559-570, 1995.

[21] G.H. Miller and P. Colella. A high-order Eulerian Godunov method for elastic-plastic flow in solids. Journal of Computational Physics, 167(1):131-176, 2001.

[22] S. Ndanou, N. Favrie, and S. Gavrilyuk. Criterion of hyperbolicity in hyperelasticity in the case of the stored energy in separable form. Journal of Elasticity, 115(1):1-25, 2014.

[23] S. Ndanou, N. Favrie, and S. Gavrilyuk. Multi-solid and multi-fluid diffuse interface model: Applications to dynamic fracture and fragmentation. Journal of Computational Physics, 295:523-555, 2015 .

[24] S. Ndanou, N. Favrie, and Gavrilyuk S.L. The piston problem in hyperelasticity with the stored energy in separable form. Mathematics and Mechanics of Solids, 2015.

[25] A López Ortega, M Lombardini, D.I. Pullin, and D.I. Meiron. Numerical simulation of elasticplastic solid mechanics using an Eulerian stretch tensor approach and HLLD Riemann solver. Journal of Computational Physics, 257:414-441, 2014.

[26] B.J. Plohr and D.H. Sharp. A conservative formulation for plasticity. Advances in Applied Mathematics, 13(4):462-493, 1992.

[27] R. Saurel and R. Abgrall. A multiphase Godunov method for compressible multifluid and multiphase flows. Journal of Computational Physics, 150(2):425-467, 1999.

[28] R. Saurel and O. Lemetayer. A multiphase model for compressible flows with interfaces, shocks, detonation waves and cavitation. Journal of Fluid Mechanics, 431:239-271, 2001.

[29] G.I. Taylor. The testing of materials at high rates of loading. J. Inst. Civil Eng, 26:486-519, 1946.

[30] G.I. Taylor. The use of flat-ended projectiles for determining dynamic yield stress. I. Theoretical considerations. In Proceedings of the Royal Society of London A: Mathematical, Physical and Engineering Sciences, volume 194, pages 289-299. The Royal Society, 1948.

[31] E.F. Toro. Riemann solvers and numerical methods for fluid dynamics: a practical introduction. Springer Science \& Business Media, 2009. 